\title{
MICROSTRUCTURE AND MECHANICAL PROPERTIES OF Mo-Nb MICROALLOYED MEDIUM MANGANESE TRIP STEEL BY CYCLIC QUENCHING
}

\author{
Chunquan Liu*, Qichun Peng, Zhengliang Xue \\ The State Key Laboratory of Refractories and Metallurgy, Key Laboratory for Ferrous \\ Metallurgy and Resources Utilization of Ministry of Education, Wuhan University of \\ Science and Technology, Wuhan 430081, China
}

Received 15.05.2019

Accepted 17.06.2019

\begin{abstract}
A novel cyclic quenching (CQ) and austenite reverse transformation (ART) was proposed for a Fe-0.25C-3.98Mn-1.22Al-0.20Si-0.19Mo-0.03Nb (wt.\%) Mo-Nb microalloyed medium-Mn TRIP steel to improve strength and ductility. The results show that after twice cyclic quenching and ART exhibited optimum comprehensive properties, characterized by an ultimate tensile strength of $838 \mathrm{MPa}$, a total elongation of $90.8 \%$, a product of strength and elongation (PSE) of $76.1 \mathrm{GPa} \cdot \%$, and the volume fraction of austenite of approximately $62 \mathrm{vol} . \%$.
\end{abstract}

Keywords: medium Mn steel; Mo-Nb microalloyed; cyclic quenching; austenite reverse transformation; austenite stability.

\section{Introduction}

In order to meet the development trend of energy saving, consumption reduction, environmental protection and safety of automobiles, it is urgent to develop a new generation of advanced high-strength steels. Therefore, it is necessary to reduce the weight of the vehicle, fuel consumption, and emissions while ensuring the safety of the vehicle [1-3]. Nowadays, medium Mn steels ( 3-11 wt.\% Mn) have become an appealing topic due to a high content of retained austenite, thus exhibiting an excellent combination of strength and elongation [4-6]. Generally, retained austenite is obtained by intercritical annealing, quenching-partitioning, reverse phase transformation annealing, etc., and the transformation induced plasticity (TRIP) effect produced by austenite deformation can significantly improve the overall mechanical properties of the steel [7-9]. It is known that retained austenite (RA) with excessive stability cannot exhibit an extensive TRIP effect,

*Corresponding author: Chunquan Liu, liuchunquan@wust.edu.cn 
which hinders the transformation of RA into martensite under continuous strain conditions, resulting in poor mechanical properties. However, RA with low stability will be deformed immediately when it is subjected to small strain, resulting in unsustainable work hardening rate of medium Mn steel [10-12]. In summary, obtaining RA with higher content and better stability is the key factor for the development of medium-Mn TRIP steel. Studying the reasonable ratio of multi-phase structure and its coordinated deformation during the deformation process is the key point of the induced phase transition.

In this work, a cyclic quenching and austenite reverse transformation (CQ-ART) process are proposed to obtain the high fraction of retained austenite in $\mathrm{Nb}-\mathrm{Mo}$ microalloyed medium-Mn TRIP steel, and its mechanical properties and plasticizing mechanism are systematically analyzed.

\section{Experimental procedure}

The ingot of steel was prepared by vacuum induction melting $(15 \mathrm{~kg})$, and the investigated alloy is a Fe-0.25C-3.98Mn-1.22Al-0.20Si-0.19Mo-0.03Nb (in wt.\%) coldrolled sheets of $1.9 \mathrm{~mm}$ in thickness. The intercritical region Ac1 and Ac3 temperatures were determined using the Thermo-Calc ${ }^{\circledR}$ and TCFE7 databases, and the values were $632{ }^{\circ} \mathrm{C}$ and $862{ }^{\circ} \mathrm{C}$, respectively. For once cyclic quenching and austenite reverse transformation (CQ-ART) sample, the sheet was water quenched after austenitization at $900{ }^{\circ} \mathrm{C}$ for $30 \mathrm{~min}$ and then intercritically annealed at $675^{\circ} \mathrm{C}$ for $1 \mathrm{~h}$, followed by air cooling to room temperature (abbreviated as CQ1-ART). For twice CQ-ART sample, the sheet was added to the same cyclic quenching heat treatment with a 10 min holding time based on CQ1. For thrice CQ-ART sample, the sheet was added to the same cyclic quenching heat treatment with a 5 min holding time based on CQ2. Finally, two sheets were intercritically annealed at $675{ }^{\circ} \mathrm{C}$ for $1 \mathrm{~h}$ before air cooling to room temperature (abbreviated as CQ2-ART and CQ3-ART, respectively). All samples were heated in a way that the muffle furnace reaches the set temperatures and then samples were put for isothermal holding, thus achieving the effect of rapid heating (as shown in Fig. 1).

Tensile tests were carried out on a microcomputer controlled electronic universal testing machine (UTM5504GD) at room temperature with a tensile rate of $3 \mathrm{~mm} / \mathrm{min}$, using tensile specimens with an original width of $12.5 \mathrm{~mm}$ and an original gauge length of $25 \mathrm{~mm}$. Microstructures of the specimens were characterized by field emission gun scanning electron microscope (Nova 400 Nano SEM) and transmission electron microscope (JEM $2100 \mathrm{~F}$ TEM). The content of retained austenite was measured by Xray diffraction analysis (XRD) (Bruker, Karlsruhe, Germany) based on the diffraction peak intensities of (200) $\alpha,(211) \alpha$, , (311) $\gamma,(200) \gamma$ and (211) $\gamma$ [13]. 


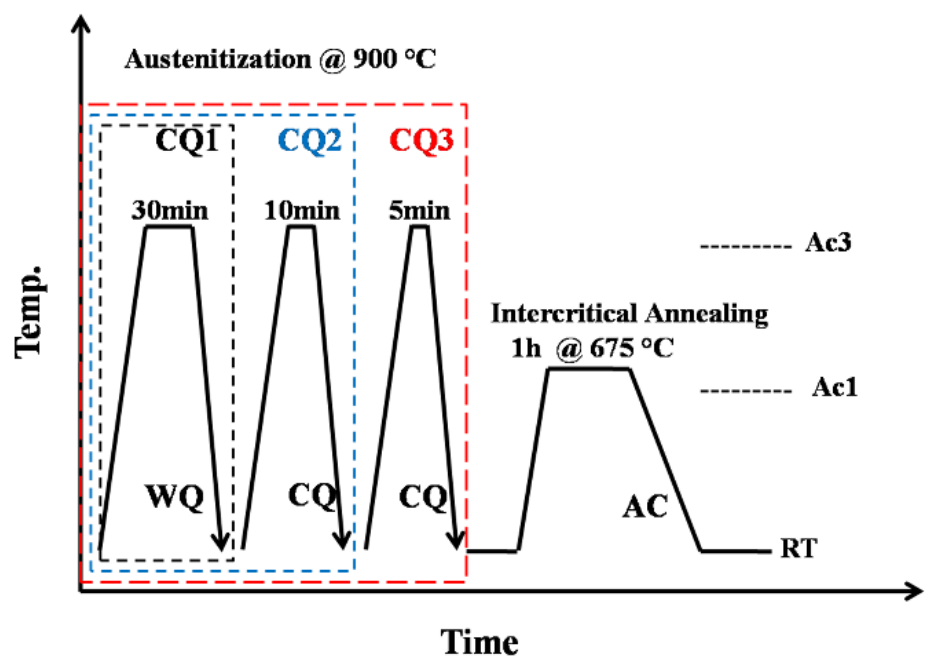

Fig. 1. Schematic of the thermo-mechanical treatment for the experimental steel (WQ: Water quenching; CQ: Cyclic quenching; RT: Room temperature).

\section{Results and discussion}

Fig. 2 a-c and $d-f$, exhibit the SEM and TEM images of CQ-ART samples, respectively. In all samples, the constituents of the microstructure mainly consist of stripshaped retained austenite (RA) and ferrite. For the CQ1-ART sample (see Fig. 2a and d), the average size of strip-shaped RA and ferrite seems to be larger than CQ2-ART and CQ3-ART samples. For the CQ2-ART and CQ3-ART sample (see Fig. 2b, e, and c, f) the microstructure mainly consists of strip-shaped RA, ferrite, and a small amount of granular austenite. The microstructure of CQ1-ART sample mainly consists of coarse strip-shaped RA and ferrite (see Fig. 2a and d), while that of CQ2-ART sample consists of relatively fine and short strip-shaped RA and ferrite (see Fig. 2b and e). The sheets were heated repeatedly and rapidly to the austenitizing temperature for a short period and then cooled rapidly so that the recrystallized austenite grains are forcibly interrupted before they grow up, and then finer lath martensite is produced during subsequent quenching process [14]. In the subsequent ART process, the recovered residual austenite tends to nucleate along the thinner lath martensite boundary. Finally, relatively small banded RA can be obtained at room temperature [15]. For the CQ3-ART sample, the microstructure is significantly smaller than the CQ1-ART sample and comparable to the CQ2-ART sample, indicating that the change in grain size after thrice cyclic quenching has been stabilized. It has been widely discussed in the literature that small grain size and $\mathrm{C} / \mathrm{Mn}$ enrichment would increase the austenite stability [16-20]. Hence, in order to clarify the mechanism of the retained austenite enhancement achieved by the CQ-ART process, it is necessary to investigate the grain size and chemical composition of the retained austenite. 

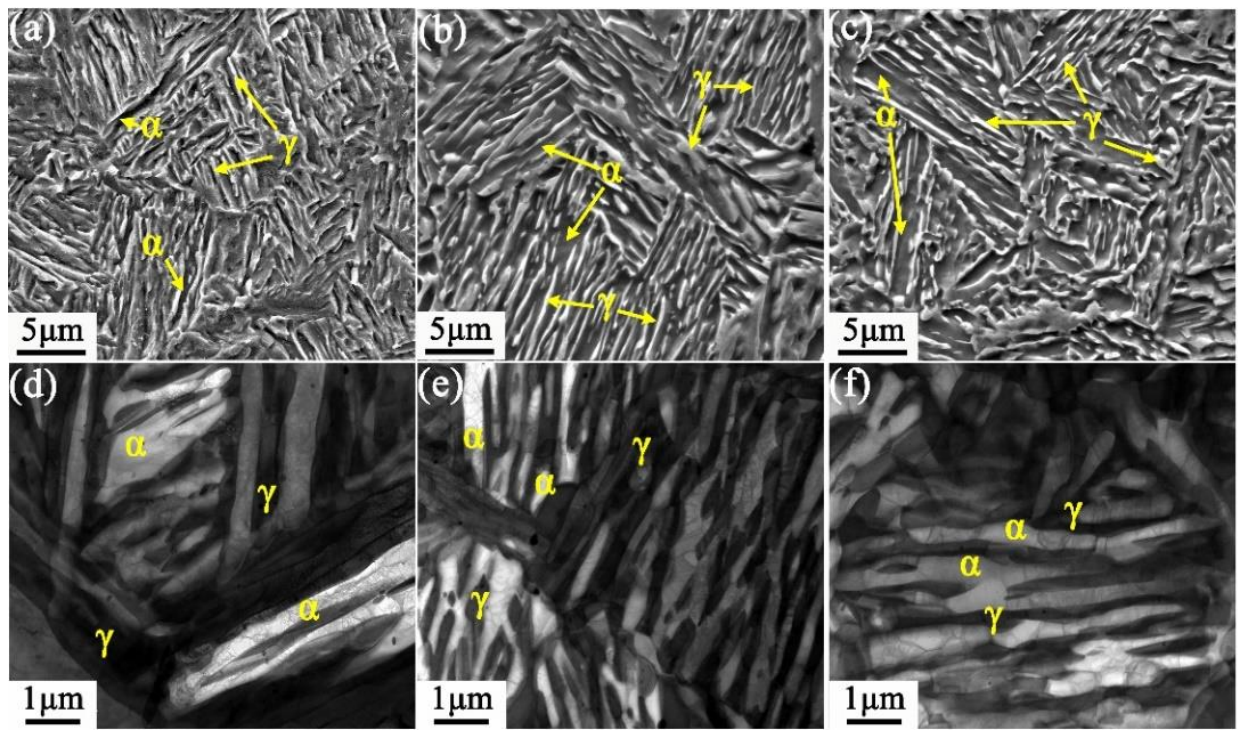

Fig. 2. SEM and TEM micrographs of $(a)-(c)$ and $(d)-(f) C Q$-ART samples, respectively, $(a, d) C Q 1-A R T$ sample, $(b, e) C Q 2-A R T$ sample and $(c, f) C Q 3-A R T$ sample ( $\gamma$ : retained austenite, $\alpha$ : ferrite).

In the process of austenite reversal transformation (ART), the austenite stabilizing elements $\mathrm{Mn}$ and $\mathrm{C}$ are distributed to austenite, while $\mathrm{Al}$ is distributed to ferrite $[1,16$, 17]. Increasing the content of $\mathrm{C}$ and $\mathrm{Mn}$ in austenite can lower the temperature of $\mathrm{Ms}$ and effectively improve the stability of austenite. The content of Mn in RA was quantitatively characterized by TEM-EDS. The average Mn concentration of RA in CQ1-ART samples was 6.16 wt.\%, while that in CQ2-ART and CQ3-ART samples was 7.31 wt.\% and 7.19 wt.\%, respectively. Fig. 3 a-c is the TEM image of CQ1-ART, CQ2-ART, and CQ3-ART samples, respectively. The corresponding Mn concentration changes in RA are shown in Fig. 3d. It is obvious that there is a significant distribution of Mn between RA and ferrite grains. In addition, granular $\left(\gamma_{\mathrm{G}}\right)$ and filmy $\left(\gamma_{\mathrm{F}}\right)$ RA was found in the samples and the Mn concentration in $\gamma_{\mathrm{G}}$ was higher than that in $\gamma_{\mathrm{F}}$. Furthermore, the Mn concentration in RA after repeated quenching is significantly higher than that in once quenching, which indicates that CQ-ART process is beneficial to the distribution of Mn to austenite. It is well known that the Mn atom belongs to substitution solute and its diffusion coefficient is much lower than those of interstitial diffusion atom, which require a long intercritical annealing time to diffuse and enrich in austenite. Cyclic quenching is equivalent to providing a longer holding time to promote the enrichment of $\mathrm{Mn}$ in austenite, reducing the $\mathrm{M}_{\mathrm{S}}$ temperature. Thus, a finer and manganese-rich martensite matrix is formed during the quenching process, and then a more stable RA was obtained at room temperature improving mechanical properties during subsequent ART. The austenite grain size of the experimental steel at room temperature was measured by Digital Micrograph software using 15 TEM images. The results show that the austenite grains with size less than 0.75 $\mu \mathrm{m}$ in CQ1-ART samples account for $69.8 \%$, while those of CQ2-ART and CQ3-ART samples account for $91.4 \%$ and $90 \%$ respectively. CQ-ART process can effectively 
reduce the austenite grain size, but the effect of grain refinement will be weakened and stabilized after three cycles of quenching.
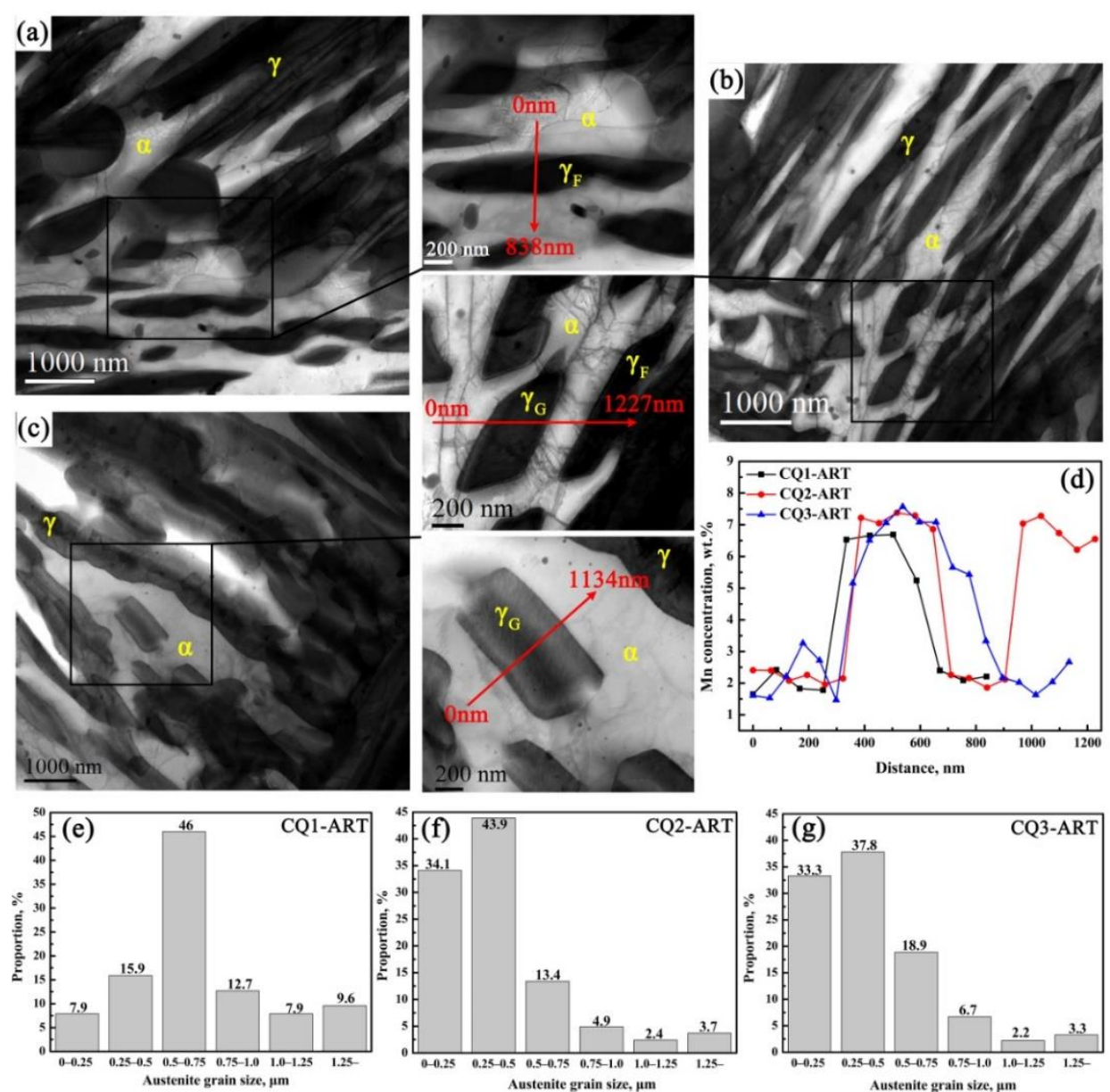

Fig. 3. TEM images of experimental steel, (a) CQ1-ART sample, (b) CQ2-ART sample, (c) CQ3-ART sample, (d) corresponding Mn concentrations measured by the TEM-EDS in $(a)-(c)$, where $\gamma, \gamma_{F}, \gamma_{G}$, and $\alpha$ refer to austenite, filmy austenite, granular austenite and ferrite, respectively. The proportion of austenite as a function of austenite size in

(e) CQ1-ART sample, (f) CQ2-ART sample and ( $g$ ) CQ3-ART sample.

The estimated austenite fraction of CQ1-ART, CQ2-ART and CQ3-ART samples before tensile tests was $\sim 49.2$ vol. $\%, \sim 62.0$ vol. $\%$ and $\sim 64.8$ vol. $\%$, respectively, which was changed to $\sim 5.9 \mathrm{vol} . \%, \sim 5.6 \mathrm{vol} . \%$ and $\sim 27.1 \mathrm{vol} . \%$ after tensile tests, respectively (see Fig. 4a and b). This result indicates that the rapid heating and CQ-ART process can significantly improve the stability of RA and thus obtain a lot of retained austenite after quenching to room temperature. The stress-strain curves and the tensile properties of experimental steels with different CQ-ART processes are summarized in Fig. 4c. The ultimate tensile strength (UTS) and total elongation (TE) increased initially and then 
decreased with the increase in the number of cyclic quenching. For CQ3-ART sample, UTS is higher than that of CQ1-ART sample, while the TE is the opposite. This means that the high stability of RA in CQ3-ART sample hinders the transformation of austenite to martensite. As a result, the ductility of CQ3-ART sample was low (TE=55.1\%), which is consistent with the conclusions in Ref. [10]. This result indicates that grain refinement contributes to the increase of strength, while high austenite conversion reflects the extensive TRIP effect, which is the guarantee of excellent properties [19]. Thus, the CQ2ART sample with a high fraction of austenite and appropriate stability exhibited optimum mechanical properties, i.e., a UTS of $838 \mathrm{MPa}$, TE of $90.8 \%$, and product of strength and elongation (PSE) of $76.1 \mathrm{GPa} \%$. By contrast, the conventional ART steels exhibited a UTS ranged from 885 to $945 \mathrm{MPa}$, TE from 13 to $28 \%$, the PSE from 11.5 to $26.5 \mathrm{GPa} \%$, and the austenite from 25.2 vol.\% to 34.2 vol.\% [20]. Furthermore, the fluctuation of work-hardening rate (see Fig. 4d) and the appearance of serrations on the engineering stress-strain curve (see Fig. 4c) are related to the release and concentration of local stress caused by TRIP effect, which was discussed in our previous study [21, 22]. The strain lengths of austenite in CQ1-ART, CQ2-ART, and CQ3-ART samples are $~ 0.447$, $\sim 0.5529$ and $\sim 0.3527$, respectively (Fig. $4 d$ ). This indicates that RA with high volume fraction and appropriate stability provided a strong guarantee for the TRIP effect in the deformation process.
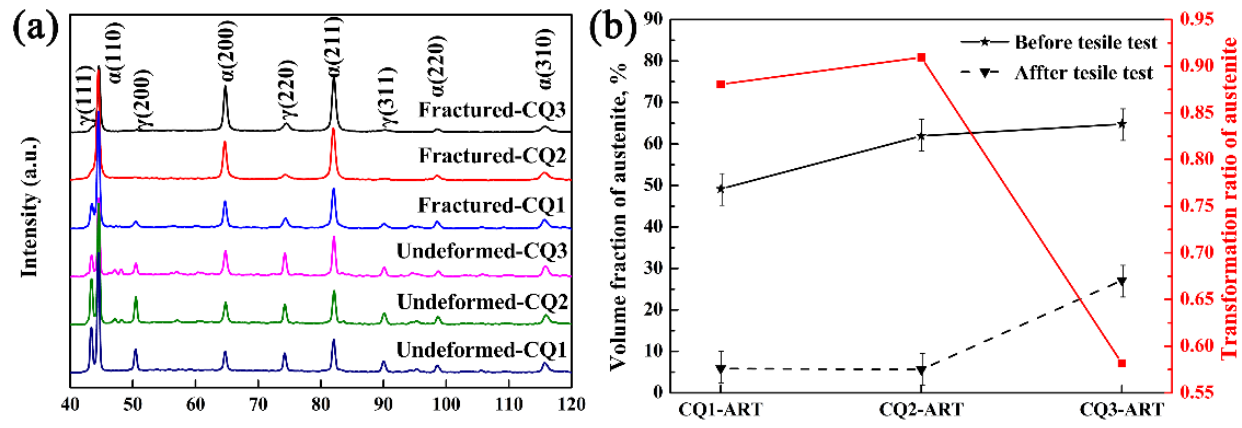

(c) 20 (deg.)
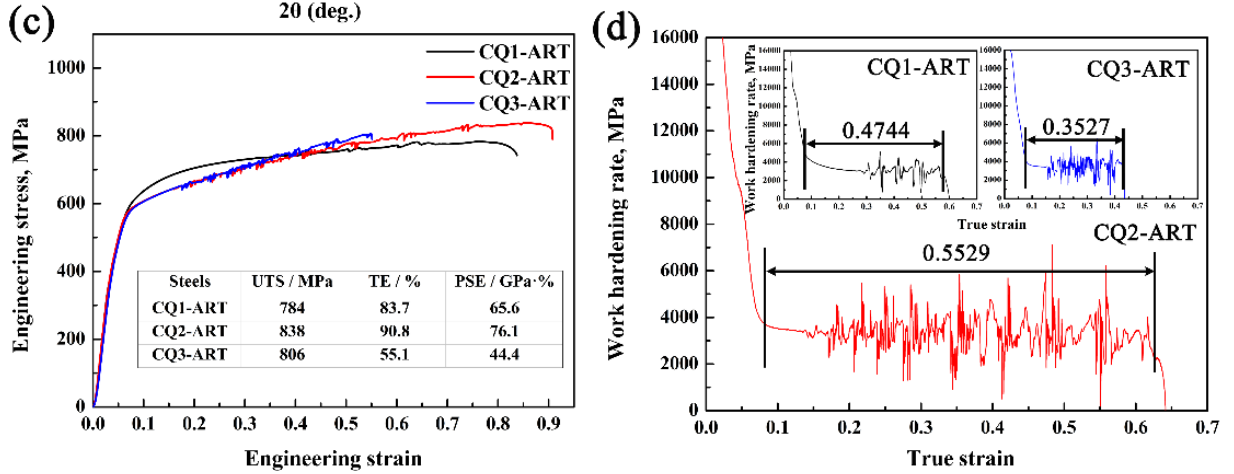

Fig. 4. (a) XRD pattern of the undeformed and fractured samples, (b) the measured austenite fraction and its transformation ratio, (c) Engineering stress-strain curves, (d) work hardening rate curves of the cycles quenching samples 


\section{Conclusion}

The stability and content of austenite in Mo-Nb microalloyed medium-Mn TRIP steel is significantly improved by a novel CQ-ART process. The cyclic quenching reverse transformation process not only refines the austenite produced by the reverse transformation but also promotes the enrichment of austenite stabilizer elements in the austenite. Therefore, the experimental steel can obtain a higher fraction and stability of RA at room temperature.

\section{Acknowledgments:}

We thank Hengyang Valin steel Tube Co, Ltd. (HYST) and China Bao Wu Wuhan Iron and Steel Group Co, Ltd. for technical assistance and financial support.

\section{References}

[1] Z.H. Cai, H. Ding, X. Xue, J. Jiang, Q.B. Xin, R.D.K. Misra: Scripta Mater, 68 (2013) 865-868.

[2] Z.H. Cai, H. Ding, R.D.K. Misra, H. Kong: Scripta Mater, 71 (2014) 5-8.

[3] B.B. He, Z.Y. Liang, M.X. Huang: Scripta Mater, 150 (2018) 134-138.

[4] H. Aydin, E. Essadiqi, I.H. Jung, S. Yue: Mater Sci Eng A, 564 (2013) 501-508.

[5] X. Li, R.B. Song, N.P. Zhou, J.J. Li: Scripta Mater, 154 (2018) 30-33.

[6] C. Wang, W.Q. Cao, J. Shi, C.X. Huang, H. Dong, Mater Sci Eng A, 562 (2013) 89-95.

[7] B. Sun, F. Fazeli, C. Scott, N. Brodusch, R. Gauvin, S. Yue: Acta Mater, 148 (2018) 249-262.

[8] J. Chen, M.Y Lv, S. Tang, Z.Y. Liu, G.D. Wang: Mater Lett, 175 (2016) 203-206.

[9] Y.G. Yang, Z.L. Mi, M. Xu, Q. Xiu, J. Li, H.T. Jiang: Mater Sci Eng A, 725 (2018) 389-397.

[10] Z.H. Cai, H.Ding, R.D.K. Misra, Z.Y. Ying: Acta Mater, 84 (2015) 229-236.

[11] L. Morsdorf, O. Jeannin, D. Barbier, M. Mitsuhara, D. Raabe, C.C. Tasan: Acta Mater, 121 (2016) 202-214.

[12] L. Zhang, X.M. Huang, Y.H. Wang, Y.B. Guo, G.Z. Dai, D.S. Li: J Mater Eng Perform (2018) 1-9.

[13] A.K. Srivastava, D. Bhattacharjee, G. Jha, N. Gope, S.B. Singh: Mater Sci Eng A 445 (2007) 549-557.

[14] R.A.Grange: ASM Trans Quart, 59 (1966) 26-48.

[15] L. Zhang, X.M. Huang, Y.H. Wang, Y.B. Guo, G.Z. Dai, D.S. Li: J Mater Eng Perform (2018) 1-9.

[16] G. Mishra, A.K. Chandan, S. Kundu: Mater Sci Eng A, 701 (2017) 319-327.

[17] K. Choi, C. Seo, H. Lee, S.K. Kim, J.H. Kwak, K.G. Chin: Scripta Mater, 63 (2010) 1028-1031.

[18] E.D. Moor, D.K. Matlock, J.G. Speer, M.J. Merwin: Scripta Mater 64 (2011) 185188.

[19] Z.P. Hu, Y.B. Xu, Y. Zou, R.D.K. Misra, D.T. Han: Materials Science and Engineering: A, 2018, 720: 1-10.

[20] Z.C. Li, H. Ding, R.D.K Misra, Z.H. Cai: Mater Sci Eng A, 682 (2017) 211-219.

[21] C.Q. Liu, Q.C. Peng, Z.L. Xue, M.M. Deng, S.J. Wang, C.W. Yang: Metals, 8 (2018) 615-630. 
[22] C.Q. Liu, Q.C. Peng, Z.L. Xue, S.J. Wang, C.W. Yang: Materials, 11 (2018) 22422253.

\author{
(c) (i) Creative Commons License
}

This work is licensed under a Creative Commons Attribution 4.0 International License. 